\title{
ON THE INFLUENCE BLOCKING MAXIMIZATION FOR MINIMIZING THE SPREADING OF FAKE INFORMATION IN SOCIAL MEDIA
}

\author{
Dema S. Alorini \\ Princess Nourah bint Abdulrahman University \\ Community College \\ Riyadh, Saudi Arabia \\ Email: dsalorini@pnu.edu.sa
}

\author{
Danda B. Rawat and Ghaida S. Alorini \\ Data Science and Cybersecurity Center (DSC2) \\ Dept. of Electrical Engineering \& Computer Science \\ Howard University, Washington, DC, USA \\ \{danda.rawat,Ghaida.Alorini\}@ @oward.edu
}

\begin{abstract}
Influence can be used to propagate the (fake or true) information in social media where a set of influential nodes (individuals) in social media can leverage their connections (e.g., followers in Tweeter) to impact others. Lately, most of the social interactions take place on-line where followers/members can get the information directly from their following accounts. Influential users can be used to propagate fake or false information to their followers. This paper analyzes social interactions in Twitter to studying the influence blocking maximization to minimize the propagation of fake information in social media by discovering influential users and their impact to spread fake/false information among their users/followers. Specifically, Greedy algorithm is studied to discover influence of spreading false/fake information among users in Twitter where malicious users could exploit influential users to gain more exposure to their malicious data. Results show that the influence of spreading fake or false information increases with the popularity of user through his/her followers and retweets. Furthermore, attributes such as number of followers, number of likes, retweets, etc. have huge impact on the influence for propagating the information in social media.
\end{abstract}

Keywords: Social Media, Fake Information, False Information, Spam, Influence Maximization/Minimization

\section{INTRODUCTION}

With the rapid increasing popularity of Social media, researchers started studying role of "Influence" for propagating information by social media users. It is a measure that influential users whose thoughts and opinions can attract other users in social media and affect their feelings and behavior. However, the impact of interaction with those influential users is not always positive. Therefore, influence can be a problem on social media.

In this paper, the focus is on studying the impact of influence for spreading of fake information or negative tweets among users. Blocking negative influence help minimize negative affect of information on Twitter. This problem is when an entity tries to block the influence distribution of another competing entity which occurs by selecting a number of seed nodes that can initiate its own influence distribution. This can be done by finding a set of influential users initiating positive influence to maximize the blocking of negative influence propagation. 
Influence maximization or minimization has been extensively studied in the state of the art work. One of the early researches is by Kempe, Kleinberg, and Tardos (Kempe, Kleinberg, and Tardos 2003) who studied the optimization problem and define it as an influence maximization problem. In addition, they defined Independent Cascade (IC) and Linear Threshold (LT). Chen, Lu, and Zhang (CHen, Lu, and Zhang 2012) studied maximizing influence by extending the IC to have a time delay of diffusion information in social media. On the other hand, Mei, Zhao and Jiang (Mei, Zhao, and Yang 2017) have studied influence maximization to maximize the impact on information spreading on Twitter which can be used for marketing purposes. Similarly, Du, Liang, Balcan, Rodriguez, Zha and Song (Du, Liang, Balcan, Gomez-Rodriguez, Zha, and Song 2016) influence maximization by identifying multiple influential users on social media to increase the adoption of products for marketing. Identifying influencers and studying their effect on other users has received widespread attention from researchers. Jendoubi, Martin, Lietard, Hend, and Boutheina (Jendoubi, Martin, Lietard, Hadji, and Yaghlane 2017) had proposed two influence maximization models to study users influence on Twitter. Borodin, Filmus, and Oren (Borodin, Filmus, and Oren 2010) analyzed influence maximization and suggested using the LT model. Other researchers tried to measure the influence value of influential users' tweets on social media. For instance, Bakshy and Mason (Bakshy, Hofman, Mason, and Watts 2011) analyzed the influence attributes of Twitter users using a dataset of $1.6 \mathrm{M}$ users. Arzkhani, Meybodi, and Rezvanian (Arazkhani, Meybodi, and Rezvanian 2019) proposed an algorithm called FC-IBM based of fuzzy clustering to find nodes for positive information on Twitter to block the influence of negative data. Similarly, the work in (Alorini, Rawat, and Alorini 2020) presents a machine Learning enabled sentiment index estimation using social media big data such as tweets. However, with the increased use of social media network the problem of influence diffusion has arisen. Two most commonly studied diffusion models are the LT and IC models. Both models require initiating a set of active nodes $A_{0}$ which starts the process of diffusion. In this approach, for a node to be active, the user has to tweet frequently. The influence is defined as a set of nodes $\mathrm{A}$ and the expected number of active users until the end of process as $\sigma(A)$. Influence Blocking Maximization (IBM) is NP-hard for both models. The modeling is defined as follows.

1. Given an input as the network as a graph $G=(\mathrm{V}, \mathrm{E})$ with $\mathrm{V}$ vertices/users and $\mathrm{E}$ links. Let us consider $k$ as a set of users/nodes which is a positive integer. The problem is to find a set of $k$ users to block the spread of negative influence as much as possible.

2. Individuals who are connected with $V$ are represented as $A$ which is a subset of $V$, that is

$$
A \subseteq V
$$

3. The maximum influence spread is $\sigma_{G}(A)$ which is NP-hard under both model LT and CT models. A greedy algorithm can be used to overcome the NP-hardness in the problem.

Twitter is a social media network that provides an opportunity for group interactions between its users. Within this network, users are connected together through the following/follower relationship. Influence maximization is a problem in social media as for the spread of rumors, new stories, propaganda and different types of information via tweets. Identifying influential users on Twitter is done by the number of followers, re-tweets, mentions and likes. Those properties measure the spread of tweets between users. The higher number of followers and re-tweets of an influential user, the higher influence of the information.

The main goal of Twitter is to share information between individuals. This data is written in form of "tweets" and can be shared with users through following/follower relationship, and hash-tags. However, the content of tweets can include malicious data, scams, or false information that can negatively impact users. Therefore, this paper studies the problem of maximizing the spread of influence within Twitter.

The remainder of this paper is organized as follows. System model is presented in Section II. Performance evaluation in Section III and conclusions in Section IV. 


\section{NETWORK MODEL AND PROBLEM FORMULATION}

Twitter is a network model $M$ on a social graph $\mathrm{G}$ of both positive and negative opinions. $M$ is modeled as the network graph between individuals $G=(V, E)$, where $V$ is the set of nodes/users in which $k$ represents a set of users in the social media and $E$ is the set of edges that represents the relationship between users such as following/followers. In addition, positive and negative edge weights are given. An edge between two users/nodes $v_{i}$ and $v_{j}$ can be represented as

$$
=e_{\left(v_{i}, v_{j}\right)}=\text { the edge connecting node } v_{i} \text { with } v_{j} .
$$

Every edge has two weights: positive $w_{u, v}^{+}$and negative $w_{u, v}^{-}$. A negative seed in influence maximization is $S_{0}^{-}$, and the positive seed is $S_{0}^{+}$, both are subset of the set $V$ as $S_{0}^{+} \subseteq V$ and $S_{0}^{-} \subseteq V$. Competitive linear threshold (CLT) which is an extension of the LT model (He, Song, Chen, and Jiang 2012b) can be used in this model where every vertex in the network model has three states: negative (-)active, positive (+)active, and inactive, and both +active and -active are not changeable once they are reached to those state. In CLT, when the positive threshold $\theta_{0}^{+}$and negative threshold $\theta_{0}^{-}$are fixed, then all randomness is fixed as well. If $S$ is a positive seed set and $N$ is a negative seed set, $v$ is positively activated at $S_{0}$ and negatively activated at $N_{0}$. And the negative influence reduction of a positive set $S$ is denoted as $\theta(s)$ (Chen, Castillo, and Lakshmanan 2013)

$$
S_{0}^{+}=\arg \max _{S_{0}^{+} \subseteq V,\left|S_{0}^{+}\right|=k} p_{-}\left(S_{0}^{+}, S_{0}^{-}\right) .
$$

This problem is NP-hard (He, Song, Chen, and Jiang 2012a). To overcome the results of NP-hard, the influence blocking maximization under CLT model is monotone and sub-modular and one can reach an approximation ratio of $1(1-1 / e-\epsilon)$ in which $\in$ is a positive number. Competitive Linear Threshold Directed Acyclic Graphs (CLDAG) with a greedy algorithm is used for influence blocking (Budak, Agrawal, and Abbadi 2011) to get the proportionates of the CLT model. This algorithm computes the negative influence from negative seed $N_{0}$ and positive seed $S$ to $v, a p^{-}(v)$. Because the greedy algorithm is very slow on a large network, the data is divided into sub-graphs, each with thousand nodes maximum. However, the testing still can be done with large dataset without the greedy algorithm. As explained earlier, the seed $S$ represents tweets. To differentiate between positive and negative weights, the propagation is defined as $p_{+}$ and $p_{-}$with values from 0 to 1 . In order to get the positive and negative weights, both propagations of each edge are multiplied. Therefore, for both propagations, the probability of a node $1-p_{+}$will not be activated.

Typical social media users does the following:

1. User registers for an account on Twitter

2. User logs into the system

3. User starts following new users and can tweet before or after following. However, the goal is to initiate with influential followers in the network. The count of influence will be measured using seeds.

4. Followers of the user view the tweet and might retweet if they like it. The tweet could include links and information, or just information. The spread of influence will be measured by using the probability of propagation.

\subsection{Independent Cascade (IC)}

IC is one of the models used in influence maximization. In this model, each edge in the graph $(\mathrm{u}, \mathrm{v})$ is connected through $\mathrm{pp}(\mathrm{u}, \mathrm{v})$, a propagation probability which is a probability of node $\mathrm{v}$ being active independently by node $\mathrm{u}$ at $\mathrm{t}+1$ while $\mathrm{u}$ is already activated at step $\mathrm{t}$. Given a Twitter as a graph for the IC model, 
and $\mathrm{k}$ as the number of followers. The influence maximization problem is to find the number of $\mathrm{k}$ nodes in the graph. The expected number of nodes activated by $\mathrm{k}$ is the influence spread. For instance, if $\mathrm{v}$ node has $\mathrm{k}$ neighbors $u_{1}, u_{2}, . ., u_{k}$, then $u_{1}$ independently activates $\mathrm{v}$ at a probability of $p_{u_{1} k}$. Kempe, Kleinberg, and Tardos (Kempe, Kleinberg, and Tardos 2003) used the greedy algorithm to ensure that the influence spread is within $(1-1 / e-\epsilon)$ where e represents the base of a natural algorithm and $\in$ represents the accuracy of the influence spread. The disadvantage of this algorithm is that it is not suitable for a large-scale network like Twitter.

\subsection{Linear Threshold (LT)}

LT is a diffusion model used in influence blocking maximization problem. In this model, $\mathrm{v}$ node has a random threshold $\theta v$ which represents the proportion of $\mathrm{v}$ neighbors that have to be active in order for $\mathrm{v}$ to be active. In LT, node $\mathrm{v}$ is influenced by its neighbors $w_{1}, w_{2}, . ., w_{k}$ based on a weight $b_{w, v}$ such that:

$$
\sum_{w, v} b_{w, v} \leq 1
$$

\subsection{Problem Statement}

Twitter is an effective online platform to advertise the products for a business with millions of active users. Advertisers can reach thousands of customers and followers instantaneously. This gives an opportunity to promote businesses and take advantage of the platform. On Twitter, almost $\% 41$ of people buy products by following an ad that precedes 30 days before (Litsa 2017). Suppose that an advertising account wants to promote a product and the target is to have influential members on the network like celebrities or famous social media influencer with hundreds or million followers. Therefore, when following these accounts, there is an opportunity to have large exposure to the advertisement. No to mention, some users just post tweets/thoughts. The problem is to find an initial, set of popular individuals with thousands or more followers. Therefore, famously known accounts can influence their followers, the followers can influence their followers and so on. This problem is called "influence maximization" which was first developed as a discrete optimization problem done by Kempe, Kleinberg, and Tardos (Kempe, Kleinberg, and Tardos 2003).

Let's assume that the tweet or advertisement is a scam or it links to a fake information and thus it is a threat and could lead to a cyber attack or significant damage. This experiment considers maximizing the blocking of negative influence through social media.

\section{PERFORMANCE EVALUATION}

\subsection{Greedy Algorithm}

The greedy algorithm is known for making the optimal choice at each stage. In influence blocking maximization, greedy is used to discover influential nodes of thoughts spreading the false information and to protect legitimate users getting fake information from malicious users.

The following greedy algorithm (GD) depends on the number of follower, re-tweets, likes, and followings. The algorithm is as follows:

Input $G=\{V, E\}$

1. for each $v$ node in $V$. 
2. for $i=1$ to $k$ do

3. $u=\arg \max _{\nu \subseteq V / S} p_{-}\left(S_{0}^{+}, S_{0}^{-}\right)$.

\subsection{Dataset}

The data in Table 1 has been extracted from Twitter using APIs. The influential user can be indicated by the date of latest tweet and number of tweets per week. In this dataset, two advertisers who use Twitter to promote their ads are examined. Both users are not verified through Twitter. Both users were considered as spammers according as in Table 1. Greedy algorithm is used to measure the spread of influence among the network. Proposed influence depends on the popularity of user through followings and followers and popularity of tweets through re-tweets and likes. The first user in Table 1 has 47,060 tweets (which is not shown in the table) and $10.8 \%$ of those tweets had an influence score depending on the re-tweets, likes and mentions. The account examined in claims of having links to download "Snap plus" and all URLs are shortened. User followed 239 users and most of the are influencers to advertise to its content. All accounts in Table 1 are not verified (i.e., they are general users) and have zero probability of being real.

\subsection{Measuring Influence}

In this paper, we analyze Twitter network as a news spreading domain and studied individual users' prospective to impact others and change their thoughts. Interaction techniques between users on Twitter involve four different activities:

1. Following Influence: it is the number of user's followers specify the size of viewers.

2. Re-tweet Influence: it is the number of re-tweets per tweet increases the number of users viewing its content.

3. Mentions Influence: it measures the number of times an account is mentioned by other accounts which specifies engagement with others' tweets.

4. Likes Influence" it measures the number of likes per tweet.

Influence attributes are used to measure the value of particular user's influence. The attributes are number followers, average number of re-tweets per tweet, and average number of likes per tweet. The fourth account in the Table 1 has five followers and all of them are fake accounts promoting the same URLs.

Table 1: Non-verified Accounts with Average Tweets, Likes, Followers and Influence Tweets.

\begin{tabular}{lcccc}
\hline User & Avg_Retweet & Avg_Likes & \#Followers & Influenced_Tweets \\
\hline @Snap_*** & 0.82 & 0.55 & 5,441 & $54 \%$ \\
\hline @ Noor02********* & 3.25 & 5.25 & 34 & $94 \%$ \\
\hline @ farfo***** & 1 & 3 & 234 & $91 \%$ \\
\hline @ mnah**** & 0.54 & 0.33 & 5 & $55 \%$ \\
\hline
\end{tabular}

The weight between two users $\mathrm{u}$ and $\mathrm{v}$ are number of re-tweet, likes, and followers. These weights $w_{1}, w_{2}, w_{3}, \ldots, w_{f}$ are used to measure the strength of relation between two users. The relation between users ensures that ' $u$ ' still receives tweets from ' $v$ '. The re-tweet weight indicate the influence weight between two users. The impact of spreading Spam by following first user directly or one of the user's followers is higher than following second user. This is determined by weight measure of the two users. In order to 
expand the study, an experiment have been applied to five hundred influential users including celebrities, politicians, businesses, and on-line bloggers.

Table 2: Top Studied Influential Accounts

\begin{tabular}{lccccc}
\hline Username & Retweets Avg. & Likes Avg. & Followers & inf & \#Mentions \\
\hline @ katyperry & $1,245.7$ & 8,552 & $108,298,219$ & $76 \%$ & 277 \\
\hline @justinbieber & $46,842.9$ & $156,332.8$ & $107,569,568$ & $83 \%$ & 469 \\
\hline @ rihanna & $11,923.8$ & $74,247.8$ & $95,073,032$ & $72 \%$ & 12647 \\
\hline @ taylorswift13 & $19,383.2$ & $108,112.6$ & $85,569,200$ & $71 \%$ & 492 \\
\hline @CNN & 149.2 & 564.8 & $43,972,649$ & $53 \%$ & 18058 \\
\hline @ladygaga & $9,971.2$ & $59,828.2$ & $80,594,804$ & $71 \%$ & 200 \\
\hline @ elonmusk & $12,460.5$ & $128,383.2$ & $30,358,314$ & $76 \%$ & 1096 \\
\hline @jack & 385.8 & $2,140.8$ & $4,368,789$ & $69 \%$ & 12352 \\
\hline @ GuyKawasaki & 3.79 & 11.2 & $1,443,810$ & $18 \%$ & 97 \\
\hline @joybauer & 2.07 & 12.1 & 153,662 & $16 \%$ & 8 \\
\hline
\end{tabular}

Table 2 presents top influential users on Twitter and their data including average number of re-tweets, average number of Likes, number of followers, influence percentage and number of mentions. The percentage of influence is an engagement comparison of this account and other accounts within the same range of followers. The comparison involves measuring number of followers, Likes, mentions and re-teweets.

All the accounts in Table 2 are verified and active. For instance, taylorswift13, posts 2.6 tweets per day (tpd) which indicates that the user is active. As seen in Table 2, the number of followers alone doesn't affect the percentage of influence. Elonmusk has less followers than other users but its percentage of influence is higher compared to other users except for @ justinbieber and @ ketyperry. However, Katyperry and Elonmusk have the highest average number of Likes compared to other users on the table and highest influence percentage as well.

On-line Spam can occur in different forms; such as, posting malicious URLs, malware, false news, or vulgar contents. Another form of Spam is the use of machine learning to produce fake reviews on spam apps and products. Then, use social bots to influence users' thoughts. Spammers follow those influential users and reply to their tweets in order to gain more exposure and views of their posts. Moreover, they use influencer's hash-tags to spread negative opinions and Spam URLs. In addition, the mention feature in Twitter is used by Spammers to spread fake news, and malicious data. The difference between hash-tags and mentions is the sign used which is '@' to mention an account and '\#' to use a hash-tag. Fig 1 shows number of

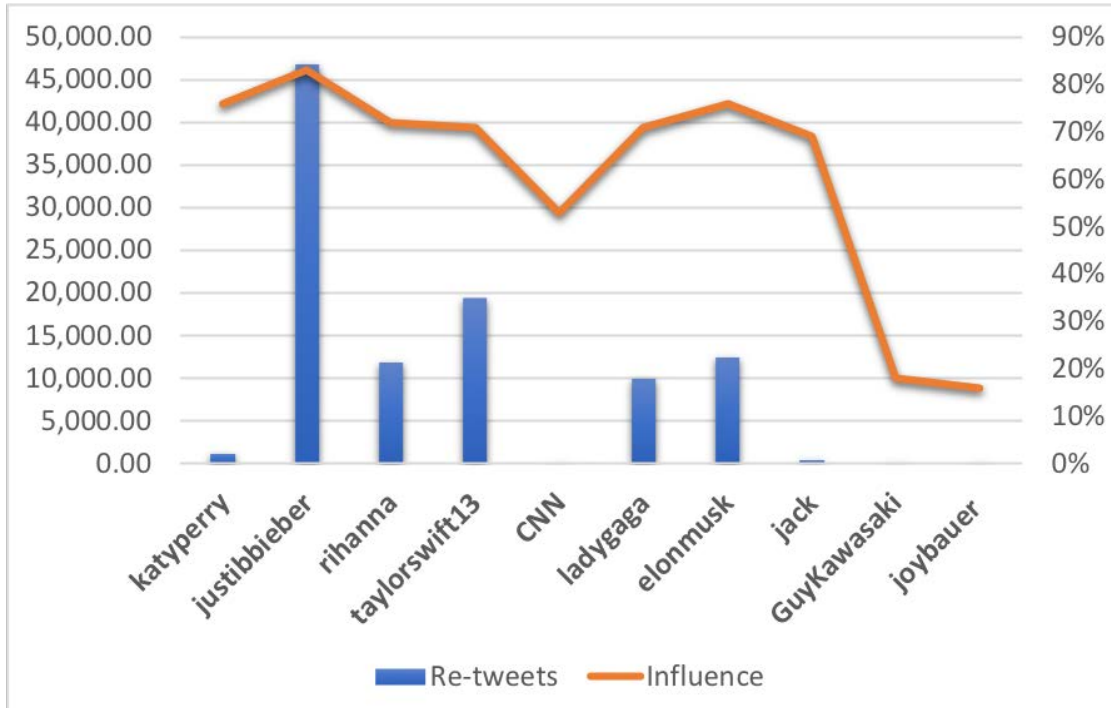

Figure 1: Re-tweets' Influence based on data in Table 2. 
re-tweets compared to the influence for each user in Table 2. It can be seen in the figure that the higher number of re-tweets, the higher number of likes an an account can receive. Similarly, Fig 2 represents the ratio of the number of likes by the influence. Fig 3 shows the number of mentions of users and how it affects the influence. The figure shows that the influence of a user doesn't depend on the number of mentions. Therefore, all attributes of an account are needed to measure percentage of influence.

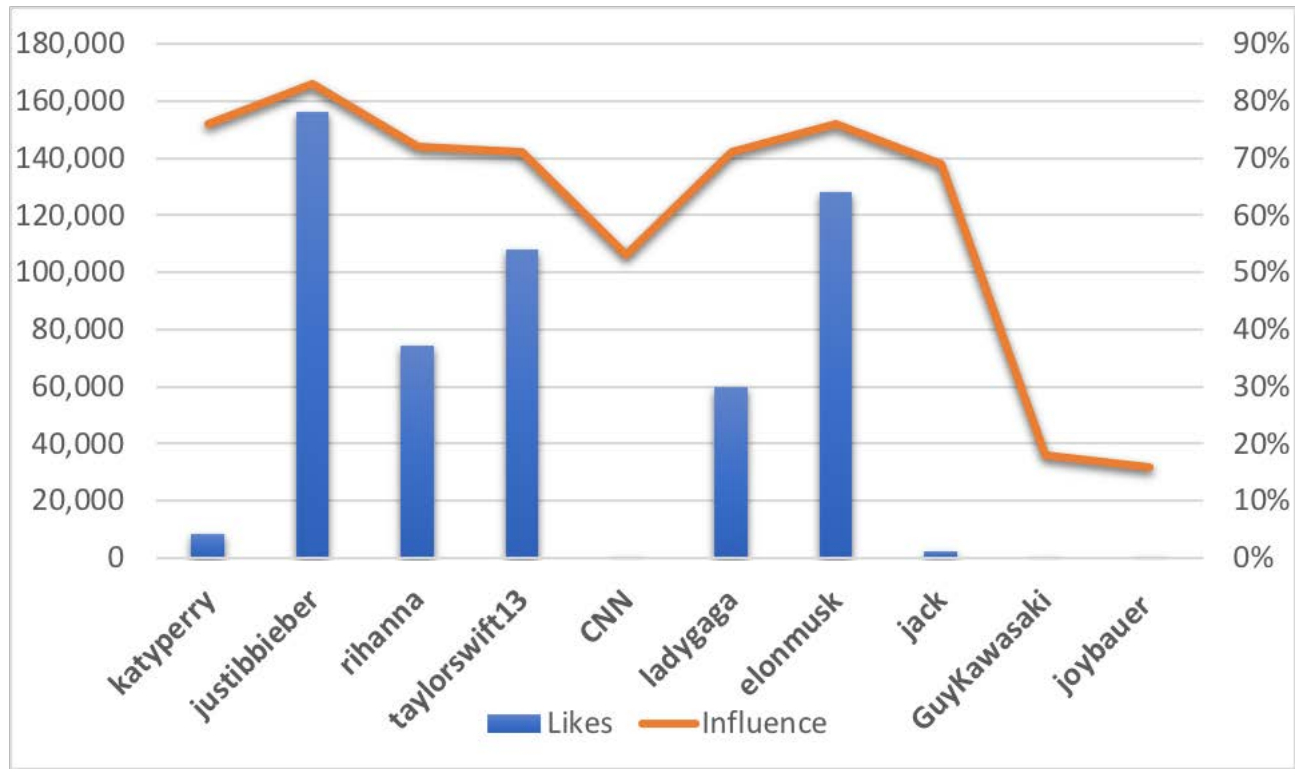

Figure 2: Likes' Influence based on data in Table 2.

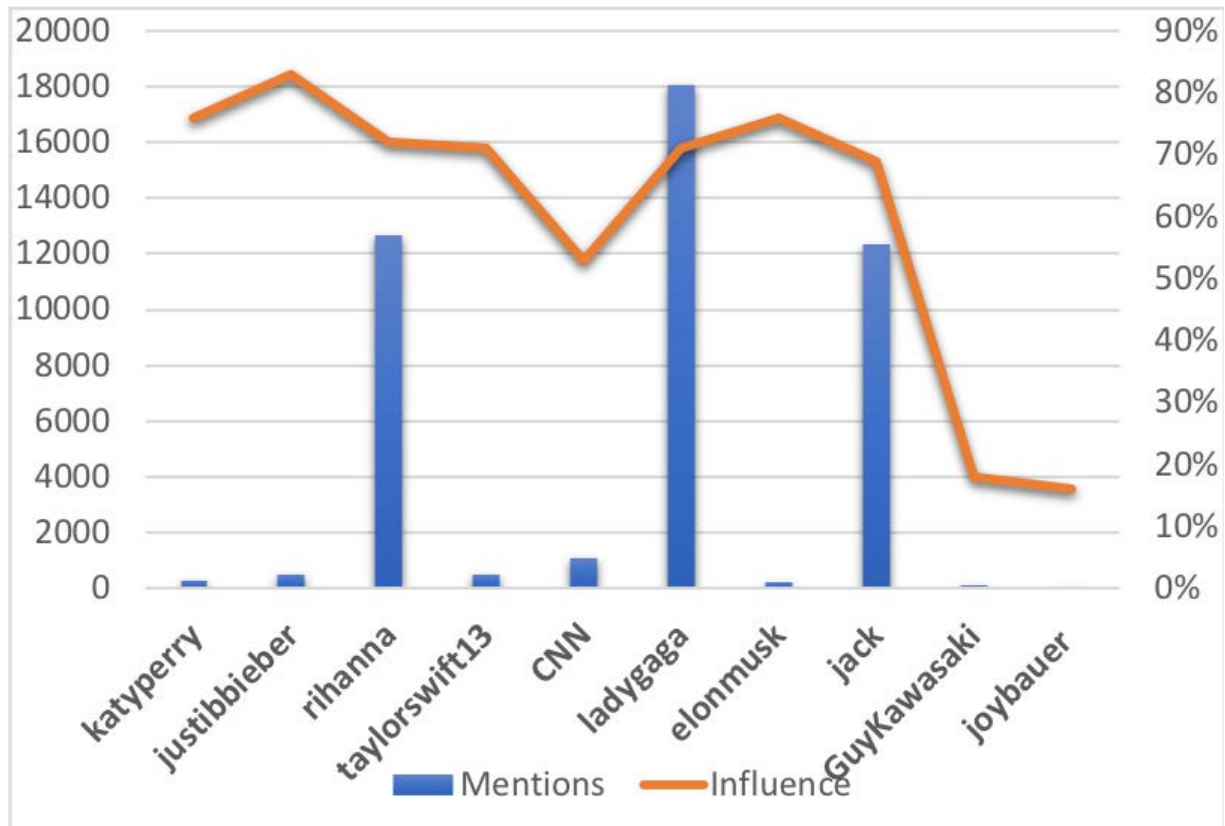

Figure 3: Mentions' Influence based on data in Table 2. 


\section{FUTURE WORK}

Twitter is a large online domain for written data and it grows rapidly. The enormous amount of data is added every day. The number of users, tweets, and hash-tags are increasing daily. Spammers come up with new ways consistently to spread malicious data and attack users. Although Twitter suspends Spam and fake accounts persistently, Spam accounts keep growing. Future work will be as follows:

1. Evaluating the proposed approach with more accounts; therefore, results would be more accurate and reliable.

2. Updating dataset as more malicious accounts and tweets grow on Twitter. It is a large corpus of data, a large number of tweets get added daily. Therefore, the data has to be updated continuously.

3. Expands the study on influence blocking maximization to block negative influence on other social media including Twitter is essential. This will help controlling the spread of misinformation and Spam through users in social media like followings, followers, Likes, Mentions and re-tweets in Tweeter.

\section{CONCLUSION AND DISCUSSIONS}

This paper has studied the influence of a user and blocking the influence to minimize the spread of fake information on Twitter by using four measures: number of followers, re-tweets, likes and mentions. Spammers come with new methods and tricks to fool people in social media. They follow influential users to expand the spread of their negative and malicious data. Influence blocking maximization is a problem of blocking the spread of fake information via social media for competing entities. In Twitter, influence blocking maximization is blocking the influence spread of tweets. When a tweet has negative influence, blocking the spread of this tweet can lower the probability of having other users viewing it. Big and known companies have their accounts verified which can be indicated by the blue mark next to their name. Therefore, people can verify real accounts that are not spam and can trust its content. Twitter recommended their users when uploading an application to check the verification mark to ensure that the account is verified. The greedy algorithm used in this paper have showed that the impact of negative influence on Twitter is high. Data of several influencers on Twitter have been extracted and tested to measure influence. Twitter attributes are used to measure influence including number of followers, re-tweets, mentions, and likes. Results showed that one attribute is not enough to predict the influence rate of an account. Attributes such as number of tweets followers, likes, re-tweets, replies, and mentions are all needed to measure influence maximization for blocking the spread of fake information .

\section{ACKNOWLEDGMENT}

The work of Dr. Dema Alorini was supported in part by the Princess Noura Bint Abdul Rahman University (PNU) at Riyadh, Saudi Arabia through her PhD scholarship. The work of Ms. Ghaida Alorini was supported in part by the Saudi Cultural Mission to the US at Washington D.C, United States. The work of Dr. Danda B. Rawat was supported in part by the U.S. National Science Foundation (NSF) grants CNS 1650831 and HRD 1828811, and by the U.S. Department of Homeland Security under grant DHS 2017-ST-062000003. However, any opinion, finding, and conclusions or recommendations expressed in this document are those of the authors and should not be interpreted as necessarily representing the official policies, either expressed or implied, of the funding agencies. 


\section{REFERENCES}

Alorini, G., D. B. Rawat, and D. Alorini. 2020, March 12 - 15. "Machine Learning Enabled Sentiment Index Estimation Using Social Media Big Data”. In IEEE SoutheastCon 2020, pp. 1-7.

Arazkhani, N., M. R. Meybodi, and A. Rezvanian. 2019. "An efficient algorithm for influence blocking maximization based on community detection". In 2019 5th International Conference on Web Research (ICWR), pp. 258-263. IEEE.

Bakshy, E., J. Hofman, W. Mason, and D. Watts. 2011, 01. "Everyone's an Influencer: Quantifying Influence on Twitter". In Proceedings of the 4th ACM International Conference on Web Search and Data Mining, WSDM 2011, pp. 65-74.

Borodin, A., Y. Filmus, and J. Oren. 2010. "Threshold Models for Competitive Influence in Social Networks". In WINE 2010, LNCS 6484, pp. 539-550.

Budak, C., D. Agrawal, and A. E. Abbadi. 2011. Limiting the spread of misinformation in social networks. Synthesis Lectures on Data Management. Morgan \& Claypool Publishers.

Chen, W., C. Castillo, and L. Lakshmanan. 2013. Information and Influence Propagation in Social Networks. Synthesis Lectures on Data Management. Morgan \& Claypool Publishers.

CHen, W., W. Lu, and N. Zhang. 2012. "Time-Critical Influence Maximization in Social Networks with Time-Delayed Diffusion Process". In Proceeding of the Twenty-Sixth AAAI Conference on Artificial Intelligence.

Du, N., Y. Liang, M.-F. Balcan, M. Gomez-Rodriguez, H. Zha, and L. Song. 2016. "Scalable Influence Maximization for Multiple Products in Continuous-Time Diffusion Networks". J. Mach. Learn. Res. vol. 18, pp. 2:1-2:45.

He, X., G. Song, W. Chen, and Q. Jiang. 2012a. "Influence Blocking Maximization in Social Networks under the Competitive Linear Threshold Model”. In Ministry of Education Key Laboratory of Machine Perception, Peking University, China.

He, X., G. Song, W. Chen, and Q. Jiang. 2012b. "Influence Blocking Maximization on Social Networks Under the Competitive Linear Threshold Model". In Proceedings of the 2012 SIAM international conference on data mining.

Jendoubi, S., A. Martin, L. Lietard, H. B. Hadji, and B. B. Yaghlane. 2017. "Two evidential data based models for influence maximization in Twitter". Knowl.-Based Syst. vol. 121, pp. 58-70.

Kempe, D., J. Kleinberg, and E. Tardos. 2003, Aug. "Maximizing the Spread of Influence through a Social Network". In Proceedings of the ninth ACM SIGKDD international conference on Knowledge discovery and data mining.

Litsa, T. 2017, Nov. "How to get started with Twitter advertising". Search Engine Watch.

Mei, Y., W. Zhao, and J. Yang. 2017. "Influence maximization on twitter: A mechanism for effective marketing campaign". In 2017 IEEE International Conference on Communications (ICC), pp. 1-6.

\section{AUTHOR BIOGRAPHIES}

DEMA S. ALORINI is an Assistant Professor of Computer Science and Information Technology at the Community College of Princess Nourah Bint Abdul Rahman University, Riyadh, Saudi Arabia. She received her $\mathrm{PhD}$ in Computer Science from Howard University, Washington, DC USA in 2018. During her PhD study, she was associated with Howard University Data Science and Cybersecurity Center (DSC2). Her research interests lie in Machine Learning and Cybersecurity. Her email address is dsalorini@pnu.edu.sa. 
DANDA B. RAWAT is an Associate Professor in the Department of Electrical Engineering and Computer Science at Howard University. Dr. Rawat is the Founding Director of the Howard University Data Science and Cybersecurity Center (DSC2), Director of Cybersecurity and Wireless Networking Innovations (CWiNs) Research Lab, Graduate Program Director of Howard-CS Graduate Programs and Director of Graduate Cybersecurity Certificate Program at Howard University, Washington, DC, USA. Dr. Rawat is engaged in research and teaching in the areas of cybersecurity, machine learning and wireless networking for emerging networked systems including cyber-physical systems, Internet-of-Things, smart cities, software defined systems and vehicular networks. He has secured over $\$ 4$ million in research funding from US National Science Foundation, US Department of Homeland Security, Department of Energy, National Nuclear Security Administration (NNSA), DoD Research Labs, Industry (Microsoft, Intel, etc.) and private Foundations. Dr. Rawat is the recipient of NSF CAREER Award in 2016, the US Air Force Research Laboratory (AFRL) Summer Faculty Visiting Fellowship in 2017, Outstanding Research Faculty Award (Award for Excellence in Scholarly Activity) at GSU in 2015, the Best Paper Awards and Outstanding PhD Researcher Award in 2009. He has delivered over 15 Keynotes and invited speeches at international conferences and workshops. Dr. Rawat received the Ph.D. degree from Old Dominion University, Norfolk, Virginia. Dr. Rawat is a Senior Member of IEEE and ACM, a member of ASEE and AAAS, and a Fellow of the Institution of Engineering and Technology (IET). His email address is Danda.Rawat@ howard.edu.

GHAIDA S. ALORINI is a PhD Student in the Department of Electrical Engineering and Computer Science at Howard University, Washington DC, USA. She is associated with Howard University Data Science and Cybersecurity Center (DSC2) for her PhD study. Her research interests include data analytics, cybersecurity and machine learning. Her email address is Ghaida.Alorini@bison.howard.edu. 